\title{
Apparatus for measuring and recording path velocity and direction characteristics of human locomotion
}

\author{
E. R. STRELOW, J. A. BRABYN, and G. R. S. CLARK \\ Department of Electrical Engineering, University of Canterbury, Christchurch, New Zealand
}

\begin{abstract}
A measuring system using electromechanical transducers and optional computer or electronic signal processing has been developed to determine characteristics of human mobility. The apparatus measures body position over a laboratory area of $80 \mathrm{~m}^{2}$ with a position error less than $2.5 \mathrm{~cm}$. The basic measuring system provides plots of path, and when used with a computer allows higher order measures based on velocity and acceleration.
\end{abstract}

The apparatus described in this paper measures and records the path of the human body during locomotion with a high level of accuracy.

The measuring system as a whole (Figure 1) comprises two mechanical sensors, which register subject position by measuring the length of a line connecting the subject to each sensor (Figure 2), photoelectric systems to convert from mechanical to electrical signals, and a digital computer to transform the signals into a two-dimensional Cartesian coordinate representation of the space within which the subject moves. Paths are printed out using an on-line plotter. If only plots of paths are required, the appropriate processing can be done by using an analog computer or analog components and a digital-to-analog converter (see below).

\section{PROBLEMS OF MOBILITY MEASUREMENT}

The term mobility describes the skills involved in walking within the normal physical environment, avoiding obstacles, and proceeding toward goals. Purposeful mobility is among the most common visually controlled human activities and involves complex perceptual as well as motor skills (see Gibson, 1958, 1966). The major thrust of research in this area has been to assist the rehabilitation of the blind (e.g., Kay, 1974, Note 1), for whom loss of vision tends to restrict normal travel. Even this research is impeded by the lack of a theoretical understanding of the basis of performance. One of the major stumbling blocks appears to be the difficulty of measuring mobility. The present report

The authors acknowledge the financial support of the University Grants Committee in establishing the mobility laboratory; appreciation is also expressed to the University of Canterbury, Department of Electrical Engineering. We wish to thank the following persons for assistance in the preparation of this article: Professor L. Kay, Dr. R. M. Hodgson, and Dr. H. R. Sirisena of the University of Canterbury; Professor R. H. Day of Monash University, and Professor I. Pollack of the University of Michigan.

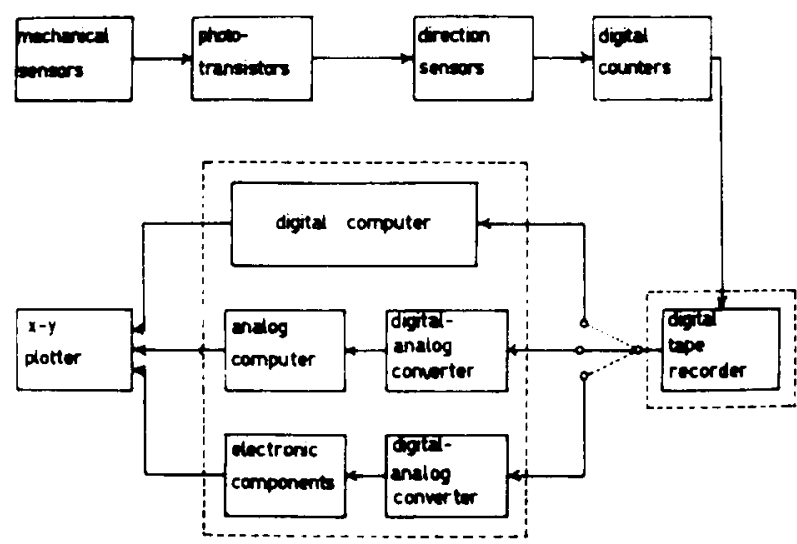

Figure 1. System block diagram showing processing options in dotted lines.

describes apparatus aimed at overcoming this deficiency.

Mobility does not easily lend itself to laboratory study, as it is difficult to measure in a manner that is both objective and convenient. Large laboratories are generally required to allow subjects to travel more than a few steps: the present apparatus is installed in a room $17 \times 11 \mathrm{~m}$. Large areas rule out the use of arrays of photocells to sense position, because the number of sensors required for more than crude spatial resolution would be quite prohibitive. At the same time, the areas are too small for conventional radar techniques. The distances involved could be resolved by ultrasound, but this technique was ruled out in our case because of likely interference with some of the aids actually studied (e.g., the binaural ultrasonic aid, Kay, 1974). But ultrasound systems are complex and expensive, as are systems to measure target position by monitoring the angles subtended at two microwave receivers from a transmitter on a moving subject.

Films and videotapes have been widely used (e.g., Armstrong, Note 2). In fact, videotape is used as a backup to the present apparatus to record the more global aspects of performance. However, these techniques 


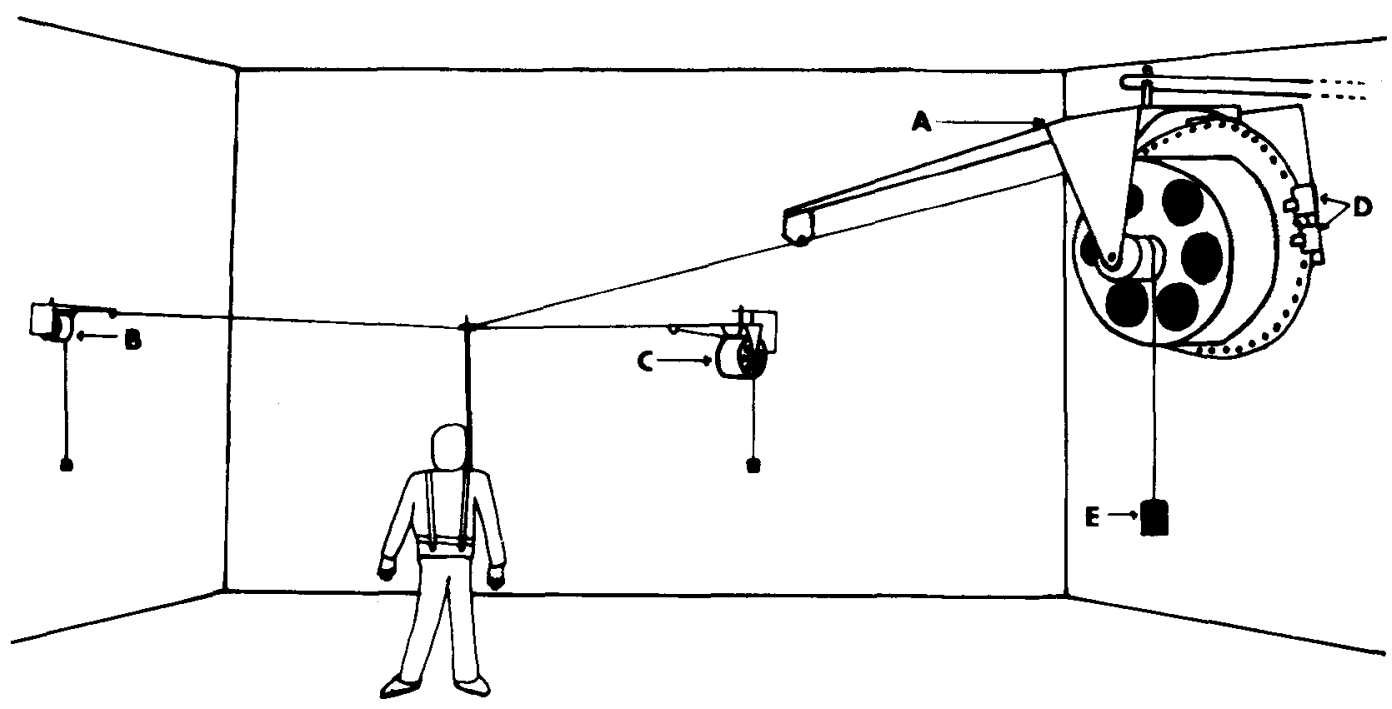

Figure 2. Drawing of mechanical sensing arrangement showing: two sensing drums ( $A$ and $B$ ) and third, balancing drum (C). Close-up of drum (A) shows photocell pair (D) and geared pulley with tensioning weight (E). The subject is shown with harness for fastening the pole to his back.

still require subsequent analysis. To measure the fine details of performance (e.g., path and velocity deviations), it is necessary to make a frame-by-frame analysis of the filmed record, which can be both tedious and inaccurate. Computer picture-processing techniques can be employed for use with cameras (Cheng, Koozekanani, \& Fatehi, 1975) but are quite complex and give very limited accuracy in covering a large area. The system described herein provides a high level of accuracy and convenience for an acceptably low level of cost and complexity. Excluding the cost of computers and analog processors, the price of electronics and materials is about $\$ 30$. The necessary mechanical labor took a skilled technician less than a week to complete.

\section{SYSTEM DESCRIPTION}

\section{Mechanical Sensors}

The position of an object in a plane is uniquely determined by its distance from two measuring points, provided the object does not cross the baseline joining these points. This principle is utilized by the apparatus described here, which effectively measures the length of two Dacron lines joining the object (the human subject) to the two measuring points (the take-up reels). Two reels are positioned in the laboratory at opposite ends of the long wall (see Figure 2). A third line is attached to another reel in the middle of the opposite wall and acts to balance the pull of the subject exerted by the two measuring drums. The three line tensions are equal under static conditions but vary when the subject accelerates because of the inertia of the drums and takeup weights used to wind up the line. The drums are grooved to guide the winding of the line and are constructed of light gauge aluminum to minimize inertia. The diameter of the drums is $10.5 \mathrm{~cm}$; the geared pulleys are $2.0 \mathrm{~cm}$ in diam. The mass of the weights $(180 \mathrm{~g})$ is calculated to give as low a value as possible, subject to accuracy constraints imposed by sag and stretch in the line. The geared pulley arrangement converts this weight to a line tension of $33.0 \mathrm{~g}$. Calculations and observations of the system in operation show it to be capable of tracking a subject who is accelerating or decelerating at $3 \mathrm{~m} / \mathrm{sec}^{2}$ in any direction, a level within the limits of the task studied. Higher accelerations can be tracked by increasing the tensioning weights, but at the cost of increasing the forces acting on the subject. The tensioning weights are kept as low as possible to minimize the likelihood that they cue the subject as to his position in the room. This would be undesirable in experiments designed to study the use of limited sensory information to judge position in the environment.

The measuring lines are attached to a circular washer that is in turn fastened to a $1-\mathrm{m}$ pole. The washer freely pivots on the tip of the pole and thus prevents the lines from tangling or winding around the pole as the subject turns. The pole is fastened to the subject's back by means of a leather harness. Wearing this harness, the directional forces generated by the mechanical coupling are undetectable by the subject over the accurate area of measurement (see below).

\section{Photoelectric Sensors and Digital Counters}

Holes $.5 \mathrm{~cm}$ in diam are drilled $1 \mathrm{~cm}$ apart along the rims of the two sensing drums. Two LED phototransistor pairs (General Electric H12A) straddle the rim of each, so that an "on" pulse is activated when a hole is detected (see Figure 2). These pulses are fed through Schmitt triggers to produce square waves. The two phototransistor pairs are so spaced around the rim that their respective square-wave trains, generated as the drum moves, will be $90 \mathrm{deg}$ out of phase. This phase 
difference reverses if the drum moves in the opposite direction. Thus, the amount of rotation of the drum is encoded by the number of pulses from one of the phototransistors, and the direction is determined by using digital logic to detect the phase difference between the two signals. This arrangement is repeated for the second sensing drum.

The direction sensing logic was realized by using two integrated-circuit flip-flops and four monostable multivibrators. The hole positions are counted by a 12-bit up-down counter formed by cascading three 4-bit updown counters. A resolution of 12 bits allows the counting of a maximum of 4,096 "hole rotations," which, given that each hole rotation corresponds to $.8 \mathrm{~cm}$, allows a line extension of about $32 \mathrm{~m}$ to be measured with a resolution of $.8 \mathrm{~cm}$.

The counter outputs from the two drums can be fed directly to a digital computer or alternatively to either an analog computer or analog components after conversion by a suitable digital-to-analog converter.

\section{Solving for Position}

To this stage of signal processing, position has been sensed in terms of distance from the two reference drums. These distances are converted to $x$ and $y$ coordinates of the actual position in the room by solving equations based on geometrical considerations (see Figure 3).

The value of " $\mathrm{a}$ " is the distance between the two measuring drums and is constant; $\mathrm{d}_{1}$ and $\mathrm{d}_{2}$ are the lengths of the two measuring lines attached to the subject. The $\mathrm{x}$ coordinate is found as follows:

$$
\begin{aligned}
d_{1}^{2} & =x^{2}+y^{2} \\
d_{2}^{2} & =(a-x)^{2}+y^{2} \\
& =a^{2}-2 a x+x^{2}+y^{2} \\
d_{2}^{2}-d_{1}^{2} & =a^{2}-2 a x \\
2 a x & =a^{2}+d_{1}^{2}-d_{2}^{2} \\
x & =\left(a^{2}+d_{1}^{2}-d_{2}^{2}\right) / 2 a
\end{aligned}
$$

The $y$ coordinate is found in terms of the $x$ coordinate as below:

$$
\begin{aligned}
y^{2} & =d_{1}^{2}-x^{2} \text { [from (1) above] } \\
y & =\sqrt{d_{1}^{2}-x^{2}}
\end{aligned}
$$

The equations for determining $\mathrm{x}$ and $\mathrm{y}$ coordinates $(5$ and 7) are repeatedly solved by an EAI 640 digital computer, in real time, at a rate of 5 samples/sec, which does not limit accuracy. Alternatively, an EAI 580 computer is used for solving these equations in an analog manner.

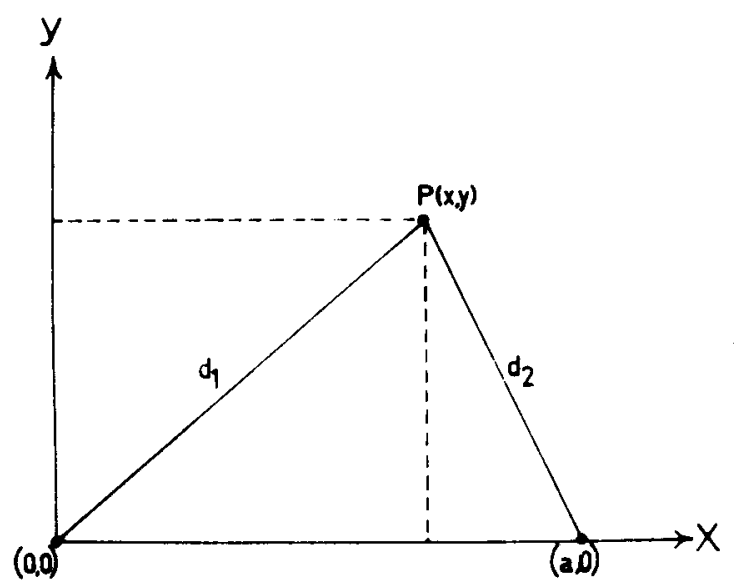

Figure 3. Geometrical arrangement. The two sensing drums are located at $(0,0)$ and $(a, 0)$.

Computers are not required if plots of position are all that is needed; the necessary processing can be done using analog components.

If the digital counter outputs are fed into two digitalto-analog converters, the resulting signals (multiplied by a scale factor) will represent $d_{1}$ and $d_{2}$, enabling solution of the equations

and

$$
\mathrm{x}=\left(\mathrm{a}^{2}+\mathrm{d}_{1}^{2}-\mathrm{d}_{2}^{2}\right) / 2 \mathrm{a}
$$

$$
y=\sqrt{d_{1}^{2}-x^{2}}
$$

by using commercially obtainable analog components. Implementation is shown schematically in Figure 4. The squaring and square root functions are performed by multipliers, while the summing and inverting functions are accomplished using operational amplifiers. For limited accuracy, integrated-circuit components (such as LM308 op-amps and XR 2208 multipliers) are acceptable; however, discrete components are preferable. The total cost of these analog components is about $\$ 40$.

Digital-to-analog conversion (DAC) can be accomplished by the use of two 10-bit Datel DAC-4910B D/A converters costing $\$ 40$ each.

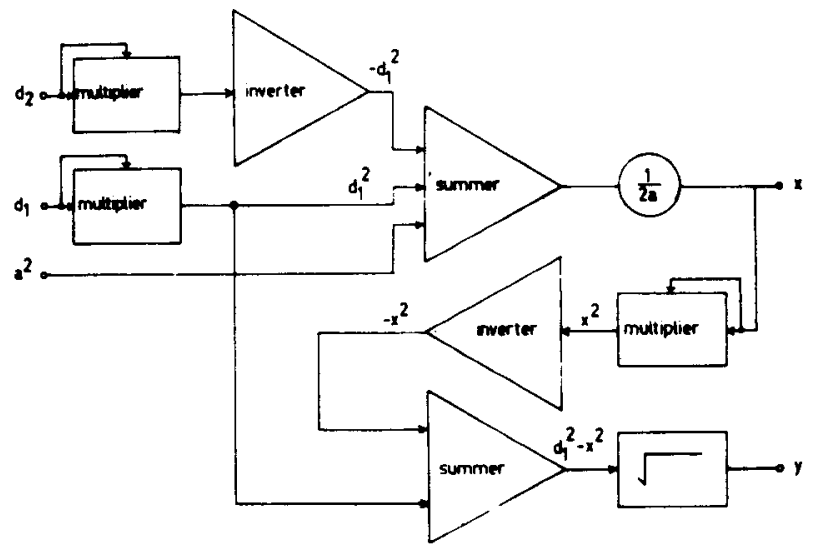

Figure 4. Diagram of analog components needed to solve for position. 
The digital computer is used so that path and velocity characteristics of performance can be analyzed mathematically. For example, in some experimental conditions, an optimum path can be specified and a measurement can be obtained of mean square deviations from this path. Irrespective of whether an optimal path can be defined, the system allows quantitative measures to be taken of performance characteristics such as smoothness and jerkiness of the path, variations in speed, and other parameters which are independent of the course the subject follows. (Approximately $16 \mathrm{~K}$ of memory is required for this type of analysis). The validation of these higher order measures of locomotion performance is one of the main research objectives and will be treated in forthcoming studies. One refinement to be added in the near future will be a digital tape recorder to allow data to be collected at any time and processed when computer time is readily available.

\section{Error Analysis}

Errors in position measurement arise from the following factors: (1) line stretch, (2) line sag, (3) hole spacing on drums, and (4) computation errors in the solution of equations. The first three of these represent errors in measurement of $d_{1}$ and $d_{2}$. Errors due to stretch variations occur only under dynamic conditions and are always less than $.16 \mathrm{~cm}$ in each line. For a constant string extension on $10 \mathrm{~m}$, the error in each line due to sag is less than $.25 \mathrm{~cm}$.

The maximum possible error due to hole spacing on drums is $.8 \mathrm{~cm}$. This can be reduced by using smaller, more closely spaced holes and cascading further counters, but the present resolution is adequate for immediate uses. Computation errors in the solution of equations are negligible when a computer is used.

A mathematical examination of errors performed by differentiating $x$ and $y$ in Equations 5 and 7 shows that overall errors, obtained by summing the four factors listed above, will always be less than $2.5 \mathrm{~cm}$ within an area of $80 \mathrm{~m}^{2}$ within the laboratory. The shape of this area corresponds roughly to a triangle with the take-up drums at the three vertices and falls within the area in which the direction of line forces would not be detectable.

\section{DETECTION OF MECHANICAL FORCES}

A psychophysical experiment was performed to determine whether the direction of forces exerted by the attached lines and measuring drums could be detected. These forces are balanced at a central position in the room, but as the subject moves toward the sides of the room, more pull will be felt in one direction than in another. It is important for the use of the apparatus in studies of space perception that the subject be unable to determine the direction of these unbalanced forces. These forces could provide a cue to his position in the room and act against experimental purposes.

\section{Method}

Measurements were taken with two subjects of the amount of force that had to be exerted through any one of the attached lines before its direction was sensed. The subject stood in the center of the room with the apparatus attached. Extra weights were then added to une of the three drums chosen at random. This procedure provided a situation comparable to that in which a subject walks toward a wall of the room and receives increasing force from one direction. Precautions were taken to insure that the subject could not gain a cue from the experimenter's actions. The subject was allowed to move freely within the central region of the room before deciding the direction of the force. Preliminary trials indicated that the likely range of weights to be added would be $60,120,180$, and $240 \mathrm{~g}$. Because of the geared pulley arrangement of the weights, these produce line tensions of $11,22,33$, and $44 \mathrm{~g}$ under static conditions.

\section{Resurts}

Chance levels of detection occurred with all weights except $240 \mathrm{~g}$, where 7 of 12 correct detections were achieved by both subjects. A binomial test showed a marginal level of statistical significance $(.05<\mathrm{p}<.10)$. Detection of forces is therefore unlikely with line tensions less than $44 \mathrm{~g}$.

Within the accurate region of measurement (i.e., where errors are less than $2.5 \mathrm{~cm}$ ), forces are less than $33 \mathrm{~g}$ with the normal weights on the drums. Thus, it can be concluded that the directional forces exerted by the mechanical attachments will be below the level of detectability within this region and will not provide cues to spatial position.

\section{APPLICATIONS}

The system has been used until now primarily in experimental studies of mobility in which subjects are given restricted spatial information via visual or auditory channels. The aim of this work has been to determine the type of spatial information needed to allow the mobile subject to negotiate various environments. The apparatus provides a record of performance in the form of a plot of the path of travel; the spatial obstacles are also marked on the plot. Errors of performance, such as passing too close to obstacles, can be measured directly from the plot. However, the use of the digital computer to assess performance by mathematically analyzing path and velocity characteristics provides both more accurate and more powerful measures. One of the major goals of the present research is the determination and validation of quantitative measures of performance. This will be achieved by comparing the performance of travelers using normal vision to that of sighted or blind travelers receiving reduced spatial information. The literature on mobility contains little in the way of such quantitative measures and the apparatus described provides a useful tool for extending quantification to this area. 


\section{REFERENCE NOTES}

1. Kay, L. Toward objective mobility evaluation: Some thoughts on a theory. American Foundation for the Blind Monograph, 1974.

2. Armstrong. J. D. Evaluation of man/machine systems in the mobility of the visually handicapped. University of Nottingham Research Report. 1973.

\section{REFERENCES}

Cheng. I.. Koozekanani, S. H., \& Fatehi. A simple computertelevision system for gait analysis. Institute of Electrical and
Electronics Engineers Transactions on Biomedical
Engineering. 1975, BME-22, No.3, 259-260.

GiBson, J. J. Visually controlled locomotion and visual orientation in animals. British Journal of Psychology, 1958, 49. 182-194.

GiBson, J. J. The senses considered as perceptual systems. London: George Allan and Unwin, 1966.

KAY, L. A sonar aid to enhance spatial perception of the blind: Engineering and evaluation. The Radio and Electronic Engineer. 1974, 44, 11.

(Received for publication January 25, 1976; revision accepted April 14. 1976.) 